\title{
The Unified Gauge Model of Four Interactions
}

\author{
Bin Liang \\ College of Science, Chongqing University of Posts and Telecommunication, Chongqing, China \\ Email: liangbin@cqupt.edu.cn
}

How to cite this paper: Liang, B. (2018) The Unified Gauge Model of Four Interactions. Journal of Modern Physics, 9, 997-1006.

https://doi.org/10.4236/jmp.2018.95062

Received: March 8, 2018

Accepted: April 22, 2018

Published: April 25, 2018

Copyright $\odot 2018$ by author and Scientific Research Publishing Inc. This work is licensed under the Creative Commons Attribution International License (CC BY 4.0).

http://creativecommons.org/licenses/by/4.0/

\begin{abstract}
We have reestablished the $S U(5)$ grand unified model according to new research results in order to clarify some misunderstandings to the model. On this basis the unified gauge model describing four interactions containing gravity has been established, and its results are consistent with experiments.

\section{Keywords}

Four Interactions, SU (5) Grand Unified Model, Grand Unified Point, Gravity, Unified Gauge Model
\end{abstract}

\section{Introduction}

Since Weinberg and Salam proposed the $S U(2) \times U(1)$ weak electromagnetic unified gauge theory in 1967 and 1968 [1], physicists have been looking for a unified description of four interactions-strong, weak, electromagnetic and gravitational interaction-using a gauge theory with only one coupling constant. They have made the long, arduous efforts towards this goal and obtained many important results. In 1974 Georgi and Glashow proposed the $S U(5)$ grand unified model on the basis of $S U_{c}(3) \times S U(2) \times U(1)$ standard model [2], but the $S U(5)$ model has been questioned because of the so-called "desert problem" and "proton decay" [3]. In addition, the attempt to establish a unified gauge theory of four interactions is also complicated by quantization of gravitational field, which remained unsolved for a long time.

So far, the following preparations have been completed in order to establish a unified gauge theory of four interactions:

1) It is proved that the dark energy is derived from the destructive interference of light [4] and quantum entanglement is an electromagnetic interaction rather than independent super-distance effect [5], so certainly there are only four inte- 
ractions in nature. 2) It is proved that gravitational field is a gauge field and its quantization and renormalization have been realized in the flat background space-time of four dimensions [6], so, a major obstacle to the establishment of unified gauge theory of four interactions has been cleared. 3) It is proved that fermions are three generations, neutrinos are massive Majorana particles, and three kinds of neutrinos are different states of the same particle [7]. So we do not have to think about the left-handed state and right-handed state of fermions. 4) It is proved that all of massive particles consist of eight gluons with fractional charge, which shows the quantization of charge [8].

With these preparations above we can establish the unified gauge theory of four interactions. First, we will reestablish the $S U(5)$ grand unified model to describe strong, weak and electromagnetic interaction according to the research results above, and explain the so-called "desert problem" and "proton decay" are misunderstandings to the $S U(5)$ model. We will then establish the unified gauge model to describe four interactions containing gravity on this basis, and yield some results consistent with experiments. We suggest readers to read the literature 7 and 8 listed at end of this article before proceeding further.

\section{The Reestablished SU(5) Model}

Since both the strong interaction and weak interaction are short-range forces, we investigate the electron-proton depth inelastic scattering [9] [10], whose course is

$$
\begin{aligned}
& e^{-}+p \rightarrow e^{-}+\Delta^{*+}(1236), \\
& \Delta^{*+}(1236) \rightarrow \pi^{+}+n \rightarrow\left(e^{+}+v_{e}\right)+\left(p+W^{-}\right) \\
& \rightarrow\left(e^{+}+v_{e}\right)+p+\left(e^{-}+v_{e}\right) \rightarrow p+2 \gamma+2 v_{e},
\end{aligned}
$$

where are no longer distinct neutrinos or antineutrinos in the equation above.

It should be explained that: 1) It is already proved that the quarks confined within nucleon are asymptotically free, namely, the deeper inside nucleon, the weaker the strong interaction between quarks. The probability density of quark distribution in nucleon is the greatest at a quarter of the radius of nucleon and equals zero at the center [7], therefore the interaction of incident electron with quarks is strongest at a quarter of the radius of nucleon and equals zero at the center. 2) From Equation (1) we see that the greater the energy of the incident electron, the greater the energy of the intermediate boson $W^{-}$, and the stronger the weak effect. 3) According to relativity, the greater the energy of the intermediate boson, the greater its mass, and the stronger the attraction between it and quarks. Therefore, it may be conceived that the intensity of four interactions can be described by the same coupling constant at a quarter of the radius of nucleon, and the position is called the grand unified point, as shown in Figure 1. The ordinate is the coupling constant, the abscissa is the distance to the center of nucleon, $\mathrm{R}$ is the radius of nucleon and the curves 1,2, 3 and 4 are strong, electromagnetic, weak and gravitational constant, respectively.

Considering each quark has the three "colors" gives the base vector 


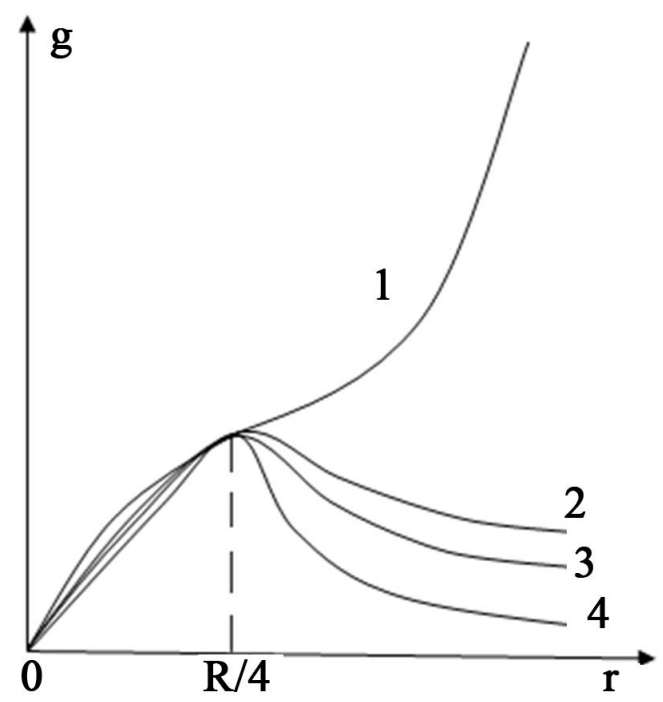

Figure 1. The grand unified point.

$$
\psi(x)=\left(\begin{array}{c}
q_{1} \\
q_{2} \\
q_{3} \\
e^{-} \\
v_{e}
\end{array}\right),
$$

where $q_{i}(i=1,2,3)$ is the same quark with different colors, $e^{-}$is electron, and $v_{e}$ is electron neutrino. Since neutrino is a massive Majorana particle, it has both left-handed and right-handed states as the same as other fermions. So, the equation above, as the base vector, can represent the left-handed or right-handed state.

The $S U(5)$ group is a 4 rank group, and its 24 generating elements are [2]

$$
\begin{aligned}
& T_{1}=\frac{1}{2}\left(\begin{array}{ccccc}
0 & 1 & 0 & 0 & 0 \\
1 & 0 & 0 & 0 & 0 \\
0 & 0 & 0 & 0 & 0 \\
0 & 0 & 0 & 0 & 0 \\
0 & 0 & 0 & 0 & 0
\end{array}\right), T_{2}=\frac{1}{2}\left(\begin{array}{ccccc}
0 & -i & 0 & 0 & 0 \\
i & 0 & 0 & 0 & 0 \\
0 & 0 & 0 & 0 & 0 \\
0 & 0 & 0 & 0 & 0 \\
0 & 0 & 0 & 0 & 0
\end{array}\right) \text {, } \\
& T_{3}=\frac{1}{2}\left(\begin{array}{ccccc}
1 & 0 & 0 & 0 & 0 \\
0 & -1 & 0 & 0 & 0 \\
0 & 0 & 0 & 0 & 0 \\
0 & 0 & 0 & 0 & 0 \\
0 & 0 & 0 & 0 & 0
\end{array}\right), T_{4}=\frac{1}{2}\left(\begin{array}{ccccc}
0 & 0 & 1 & 0 & 0 \\
0 & 0 & 0 & 0 & 0 \\
1 & 0 & 0 & 0 & 0 \\
0 & 0 & 0 & 0 & 0 \\
0 & 0 & 0 & 0 & 0
\end{array}\right), \\
& T_{5}=\frac{1}{2}\left(\begin{array}{ccccc}
0 & 0 & -i & 0 & 0 \\
0 & 0 & 0 & 0 & 0 \\
i & 0 & 0 & 0 & 0 \\
0 & 0 & 0 & 0 & 0 \\
0 & 0 & 0 & 0 & 0
\end{array}\right), T_{6}=\frac{1}{2}\left(\begin{array}{ccccc}
0 & 0 & 0 & 0 & 0 \\
0 & 0 & 1 & 0 & 0 \\
0 & 1 & 0 & 0 & 0 \\
0 & 0 & 0 & 0 & 0 \\
0 & 0 & 0 & 0 & 0
\end{array}\right) \text {, }
\end{aligned}
$$




$$
\begin{aligned}
& T_{7}=\frac{1}{2}\left(\begin{array}{ccccc}
0 & 0 & 0 & 0 & 0 \\
0 & 0 & -i & 0 & 0 \\
0 & i & 0 & 0 & 0 \\
0 & 0 & 0 & 0 & 0 \\
0 & 0 & 0 & 0 & 0
\end{array}\right), T_{8}=\frac{1}{2 \sqrt{3}}\left(\begin{array}{ccccc}
1 & 0 & 0 & 0 & 0 \\
0 & 1 & 0 & 0 & 0 \\
0 & 0 & -2 & 0 & 0 \\
0 & 0 & 0 & 0 & 0 \\
0 & 0 & 0 & 0 & 0
\end{array}\right), \\
& T_{9}=\frac{1}{2}\left(\begin{array}{ccccc}
0 & 0 & 0 & 1 & 0 \\
0 & 0 & 0 & 0 & 0 \\
0 & 0 & 0 & 0 & 0 \\
1 & 0 & 0 & 0 & 0 \\
0 & 0 & 0 & 0 & 0
\end{array}\right), T_{10}=\frac{1}{2}\left(\begin{array}{ccccc}
0 & 0 & 0 & -i & 0 \\
0 & 0 & 0 & 0 & 0 \\
0 & 0 & 0 & 0 & 0 \\
i & 0 & 0 & 0 & 0 \\
0 & 0 & 0 & 0 & 0
\end{array}\right), \\
& T_{11}=\frac{1}{2}\left(\begin{array}{ccccc}
0 & 0 & 0 & 0 & 1 \\
0 & 0 & 0 & 0 & 0 \\
0 & 0 & 0 & 0 & 0 \\
0 & 0 & 0 & 0 & 0 \\
1 & 0 & 0 & 0 & 0
\end{array}\right), \quad T_{12}=\frac{1}{2}\left(\begin{array}{ccccc}
0 & 0 & 0 & 0 & -i \\
0 & 0 & 0 & 0 & 0 \\
0 & 0 & 0 & 0 & 0 \\
0 & 0 & 0 & 0 & 0 \\
i & 0 & 0 & 0 & 0
\end{array}\right), \\
& T_{13}=\frac{1}{2}\left(\begin{array}{ccccc}
0 & 0 & 0 & 0 & 0 \\
0 & 0 & 0 & 0 & 1 \\
0 & 0 & 0 & 0 & 0 \\
0 & 0 & 0 & 0 & 0 \\
0 & 1 & 0 & 0 & 0
\end{array}\right), T_{14}=\frac{1}{2}\left(\begin{array}{ccccc}
0 & 0 & 0 & 0 & 0 \\
0 & 0 & 0 & 0 & -i \\
0 & 0 & 0 & 0 & 0 \\
0 & 0 & 0 & 0 & 0 \\
0 & i & 0 & 0 & 0
\end{array}\right), \\
& T_{15}=\frac{1}{2 \sqrt{6}}\left(\begin{array}{ccccc}
1 & 0 & 0 & 0 & 0 \\
0 & 1 & 0 & 0 & 0 \\
0 & 0 & 1 & 0 & 0 \\
0 & 0 & 0 & -3 & 0 \\
0 & 0 & 0 & 0 & 0
\end{array}\right), T_{16}=\frac{1}{2}\left(\begin{array}{ccccc}
0 & 0 & 0 & 0 & 0 \\
0 & 0 & 0 & 1 & 0 \\
0 & 0 & 0 & 0 & 0 \\
0 & 1 & 0 & 0 & 0 \\
0 & 0 & 0 & 0 & 0
\end{array}\right) \text {, } \\
& T_{17}=\frac{1}{2}\left(\begin{array}{ccccc}
0 & 0 & 0 & 0 & 0 \\
0 & 0 & 0 & -i & 0 \\
0 & 0 & 0 & 0 & 0 \\
0 & i & 0 & 0 & 0 \\
0 & 0 & 0 & 0 & 0
\end{array}\right), T_{18}=\frac{1}{2}\left(\begin{array}{ccccc}
0 & 0 & 0 & 0 & 0 \\
0 & 0 & 0 & 0 & 0 \\
0 & 0 & 0 & 1 & 0 \\
0 & 0 & 1 & 0 & 0 \\
0 & 0 & 0 & 0 & 0
\end{array}\right), \\
& T_{19}=\frac{1}{2}\left(\begin{array}{ccccc}
0 & 0 & 0 & 0 & 0 \\
0 & 0 & 0 & 0 & 0 \\
0 & 0 & 0 & -i & 0 \\
0 & 0 & i & 0 & 0 \\
0 & 0 & 0 & 0 & 0
\end{array}\right), T_{20}=\frac{1}{2}\left(\begin{array}{ccccc}
0 & 0 & 0 & 0 & 0 \\
0 & 0 & 0 & 0 & 0 \\
0 & 0 & 0 & 0 & 1 \\
0 & 0 & 0 & 0 & 0 \\
0 & 0 & 1 & 0 & 0
\end{array}\right), \\
& T_{21}=\frac{1}{2}\left(\begin{array}{ccccc}
0 & 0 & 0 & 0 & 0 \\
0 & 0 & 0 & 0 & 0 \\
0 & 0 & 0 & 0 & -i \\
0 & 0 & 0 & 0 & 0 \\
0 & 0 & i & 0 & 0
\end{array}\right), T_{22}=\frac{1}{2}\left(\begin{array}{ccccc}
0 & 0 & 0 & 0 & 0 \\
0 & 0 & 0 & 0 & 0 \\
0 & 0 & 0 & 0 & 0 \\
0 & 0 & 0 & 0 & 1 \\
0 & 0 & 0 & 1 & 0
\end{array}\right),
\end{aligned}
$$




$$
T_{23}=\frac{1}{2}\left(\begin{array}{ccccc}
0 & 0 & 0 & 0 & 0 \\
0 & 0 & 0 & 0 & 0 \\
0 & 0 & 0 & 0 & 0 \\
0 & 0 & 0 & 0 & -i \\
0 & 0 & 0 & i & 0
\end{array}\right), T_{24}=\frac{1}{2 \sqrt{10}}\left(\begin{array}{ccccc}
1 & 0 & 0 & 0 & 0 \\
0 & 1 & 0 & 0 & 0 \\
0 & 0 & 1 & 0 & 0 \\
0 & 0 & 0 & 1 & 0 \\
0 & 0 & 0 & 0 & -4
\end{array}\right)
$$

where $T_{3}, T_{8}, T_{15}, T_{24}$ are four diagonal matrices. Every of 24 matrices is Hermitian without trace and orthogonal normalized as

$$
\operatorname{Tr}\left(T_{i} T_{j}\right)=\frac{1}{2} \delta_{i j} .
$$

According to the gauge theory, there are 24 gauge fields $A_{i}(x)(i=1,2, \cdots, 24)$ corresponding to the 24 generated elements above. We define the total gauge field $A_{\mu}(x)(\mu=0,1,2,3)$ as

$$
\frac{1}{\sqrt{2}} A_{\mu}(x)=\sum_{i=1}^{24} T_{i} A_{i}(x) .
$$

Substituting the Equation (3) into the equation above gives

$$
A_{\mu}(x)=\left(\begin{array}{ccccc}
S_{11} & S_{12} & S_{13} & \bar{X}_{1} & \bar{W}_{1} \\
S_{21} & S_{22} & S_{23} & \bar{X}_{2} & \bar{W}_{2} \\
S_{31} & S_{32} & S_{33} & \bar{X}_{3} & \bar{W}_{3} \\
X_{1} & X_{1} & X_{3} & X_{0} & W^{+} \\
W_{1} & W_{2} & W_{3} & W^{-} & Z_{0}
\end{array}\right)
$$

where (the subscript $\mu$ of each matrix element has been omitted)

$$
\begin{aligned}
& S_{11}=\frac{1}{\sqrt{2}}\left(A_{3}+\frac{1}{\sqrt{10}} A_{24}\right)+\frac{1}{\sqrt{6}}\left(A_{8}+\frac{1}{\sqrt{2}} A_{15}\right), \quad S_{12}=\frac{1}{\sqrt{2}}\left(A_{1}-i A_{2}\right), \\
& S_{13}=\frac{1}{\sqrt{2}}\left(A_{4}-i A_{5}\right), \quad S_{21}=\frac{1}{\sqrt{2}}\left(A_{1}+i A_{2}\right), \\
& S_{22}=\frac{1}{\sqrt{2}}\left(-A_{3}+\frac{1}{\sqrt{10}} A_{24}\right)+\frac{1}{\sqrt{6}}\left(A_{8}+\frac{1}{\sqrt{2}} A_{15}\right), \quad S_{23}=\frac{1}{\sqrt{2}}\left(A_{6}-i A_{7}\right) \text {, } \\
& S_{31}=\frac{1}{\sqrt{2}}\left(A_{4}+i A_{5}\right), \quad S_{32}=\frac{1}{\sqrt{2}}\left(A_{6}+i A_{7}\right), \\
& S_{33}=-\frac{2}{\sqrt{6}} A_{8}+\frac{1}{\sqrt{2}}\left(\frac{1}{\sqrt{6}} A_{15}+\frac{1}{\sqrt{10}} A_{24}\right) \text {, } \\
& X_{1}=\frac{1}{\sqrt{2}}\left(A_{9}+i A_{10}\right), \quad X_{2}=\frac{1}{\sqrt{2}}\left(A_{16}+i A_{17}\right), \quad X_{3}=\frac{1}{\sqrt{2}}\left(A_{18}+i A_{19}\right) \text {, } \\
& \bar{X}_{1}=\frac{1}{\sqrt{2}}\left(A_{9}-i A_{10}\right), \quad \bar{X}_{2}=\frac{1}{\sqrt{2}}\left(A_{16}-i A_{17}\right), \quad \bar{X}_{3}=\frac{1}{\sqrt{2}}\left(A_{18}-i A_{19}\right) \text {, } \\
& W_{1}=\frac{1}{\sqrt{2}}\left(A_{11}+i A_{12}\right), \quad W_{2}=\frac{1}{\sqrt{2}}\left(A_{13}+i A_{14}\right), \quad W_{3}=\frac{1}{\sqrt{2}}\left(A_{20}+i A_{21}\right) \text {, } \\
& \bar{W}_{1}=\frac{1}{\sqrt{2}}\left(A_{11}-i A_{12}\right), \quad \bar{W}_{2}=\frac{1}{\sqrt{2}}\left(A_{13}-i A_{14}\right), \quad \bar{W}_{3}=\frac{1}{\sqrt{2}}\left(A_{20}-i A_{21}\right) \text {, }
\end{aligned}
$$




$$
\begin{array}{r}
W^{+}=\frac{1}{\sqrt{2}}\left(A_{22}-i A_{23}\right), W^{-}=\frac{1}{\sqrt{2}}\left(A_{22}+i A_{23}\right), \\
X_{0}=\frac{W}{\sqrt{2}}+\frac{3 B}{\sqrt{30}}, Z_{0}=-\frac{W}{\sqrt{2}}+\frac{3 B}{\sqrt{30}}, \\
\text { and } W=-\frac{3}{2 \sqrt{6}} A_{15}+\frac{5}{2 \sqrt{10}} A_{24}, \quad B=-\frac{\sqrt{15}}{2}\left(\frac{A_{15}}{\left.\sqrt{6}+\frac{A_{24}}{\sqrt{10}}\right) .}\right.
\end{array}
$$

According to the gauge theory, the Lagrange density of gauge invariant fermions field is

$$
L_{f}=i \bar{\psi} \gamma^{\mu} D_{\mu} \psi
$$

where

$$
D_{\mu} \psi=\left(\partial_{\mu}-i g_{5} A_{\mu}(x)\right) \psi,
$$

and the $g_{5}$ is the unified coupling constant. Substituting the Equation (6) into the Equation (8) gives the interactional Lagrange density of fermions with the gauge field:

$$
\begin{aligned}
& L_{i}=g_{5} \bar{\psi} \gamma^{\mu} A_{\mu} \psi=g_{5} \bar{\psi} \hat{A}_{\mu} \psi \\
& =g_{5}\left(\bar{q}_{1} \hat{S}_{11} q_{1}+\bar{q}_{1} \hat{S}_{12} q_{2}+\bar{q}_{1} \hat{S}_{13} q_{3}+\bar{q}_{2} \hat{S}_{21} q_{1}+\bar{q}_{2} \hat{S}_{22} q_{2}+\bar{q}_{2} \hat{S}_{23} q_{3}+\bar{q}_{3} \hat{S}_{31} q_{1}+\bar{q}_{3} \hat{S}_{32} q_{2}\right. \\
& \left.+\bar{q}_{3} \hat{S}_{33} q_{3}\right)+g_{5}\left(\bar{q}_{1} \hat{\bar{X}}_{1} e^{-}+\bar{q}_{2} \hat{\bar{X}}_{2} e^{-}+\bar{q}_{3} \hat{\bar{X}}_{3} e^{-}+e^{+} \hat{X}_{1} q_{1}+e^{+} \hat{X}_{2} q_{2}+e^{+} \hat{X}_{3} q_{3}+e^{+} \hat{X}_{0} e^{-}\right) \\
& +g_{5}\left(\bar{q}_{1} \hat{\bar{W}}_{1} v+\bar{q}_{2} \hat{\bar{W}}_{2} v+\bar{q}_{3} \hat{\bar{W}}_{3} v+v \hat{W}_{1} q_{1}+v \hat{W}_{2} q_{2}+v \hat{W}_{3} q_{3}+e^{+} \hat{W}^{+} v+v \hat{W}^{-} e^{-}+v \hat{Z}_{0} v\right)
\end{aligned}
$$

The subscript $e$ of neutrino has been omitted in the equation above. Since leptons don't take part in strong interaction and neutrinos take part only in weak interaction, the nine terms in the first bracket above describe the strong interaction between valence quarks and sea quarks, the seven terms in the second bracket describe the electromagnetic interaction between particles, and the nine terms in the third bracket describe the weak interaction.

In the past people thought that the terms shaped like $e^{+} \hat{X}_{i} q_{i}, v \hat{W}_{i} q_{i}$ make quarks become leptons and cause the decay of proton, but this is a misunderstanding. We know that the mass $(5-8.5 \mathrm{MeV})$ of quark $d$ is greater than the mass $(1.5-4.5 \mathrm{MeV})$ of quark $u$. So, if the proton $p$ (uud) decays, it could only be that quark $d$ decays into quark $u$, namely the proton $p(u u d)$ gets into the resonant state $\Delta^{++}(u и u)$ with higher energy, but this is excitation rather than decay. In fact, the terms shaped like $e^{+} \hat{X}_{i} q_{i}, v \hat{W}_{i} q_{i}$ only show the existence of electromagnetic and weak interaction between quark and lepton, and the physical meaning depends on the specific physical process and cannot be generalized.

Since the seven terms in the second bracket describe the electromagnetic interaction between particles, and photons have no mass, so the medium particle whose mass is up to $10^{14} \mathrm{GeV}$ doesn't exist. Thus, the so-called "desert problem", i.e. there is no physical effect from level $10^{2} \mathrm{GeV}$ to $10^{14} \mathrm{GeV}$, has been avoided. 


\section{Mass of Intermediate Boson}

There is no mass term in the Equation (10) in order to satisfy the gauge invariance, but the intermediate bosons $W^{+}, W^{-}, Z_{0}$ and fermions are massive. The Higgs mechanism can give masses to these particles [2].

The Lagrange density of 5-dementional Higgs field $\varphi$ is

$$
L_{\varphi}=\left(D^{\mu} \varphi\right)_{\alpha}^{+}\left(D_{\mu} \varphi\right)^{\alpha}-V(\varphi),
$$

where $\alpha=1,2,3,4,5$, and the potential energy

$$
V(\varphi)=-\frac{1}{2} \mu^{2} \varphi^{+} \varphi+\frac{1}{4} \lambda\left(\varphi^{+} \varphi\right)^{2}
$$

has the minimum value at $\left\langle\varphi^{+} \varphi\right\rangle_{0}=\frac{\mu^{2}}{\lambda}$. The average value of $\varphi$ in the vacuum is

$$
\varphi_{0}=\left(\begin{array}{c}
0 \\
0 \\
0 \\
0 \\
v / \sqrt{2}
\end{array}\right),
$$

where $v^{2}=2 \mu^{2} / \lambda$. The Lagrange density in the Equation (11) is plainly gauge invariant, and

$$
\begin{aligned}
& \left(D^{\mu} \varphi\right)_{\alpha}^{+}\left(D_{\mu} \varphi\right)^{\alpha}=\left(\partial^{\mu} \varphi_{\alpha}^{+}+\frac{i}{\sqrt{2}} g_{5}\left(A_{\mu}^{+}\right)_{\alpha}^{\beta} \varphi_{\beta}^{+}\right)\left(\partial_{\mu} \varphi^{\alpha}-\frac{i}{\sqrt{2}} g_{5}\left(A_{\mu}\right)_{\beta}^{\alpha} \varphi^{\beta}\right) \\
& =\left(\partial^{\mu} \varphi_{\alpha}^{+}\right)\left(\partial_{\mu} \varphi^{\alpha}\right)-\frac{i}{\sqrt{2}} g_{5}\left[\left(\partial^{\mu} \varphi_{\alpha}^{+}\right)\left(A_{\mu}\right)_{\beta}^{\alpha} \varphi^{\beta}-\left(A_{\mu}^{+}\right)_{\alpha}^{\beta} \varphi_{\beta}^{+} \partial_{\mu} \varphi^{\alpha}\right] \\
& +\frac{1}{2} g_{5}^{2}\left(A_{\mu}^{+}\right)_{\alpha}^{\beta} \varphi_{\beta}^{+}\left(A_{\mu}\right)_{\beta}^{\alpha} \varphi^{\beta} .
\end{aligned}
$$

Considering the translational transformation:

$$
\varphi=\varphi_{0}+\varphi^{\prime},
$$

where $\varphi_{0}^{\alpha}=\frac{v}{\sqrt{2}} \delta_{\alpha 5}$ as shown in Equation (13). Taking the unitary gauge $\varphi_{\alpha}^{\prime}=\frac{\eta}{\sqrt{2}} \delta_{\alpha 5}$ gives

$$
\begin{aligned}
\left(D^{\mu} \varphi\right)_{\alpha}^{+}\left(D_{\mu} \varphi\right)^{\alpha}= & \frac{1}{2} \partial^{\mu} \eta \partial_{\mu} \eta+\frac{1}{2} g_{5}^{2} v \eta\left(A_{\mu}^{+}\right)_{\alpha}^{5}\left(A^{\mu}\right)_{5}^{\alpha}+\frac{1}{4} g_{5}^{2} \eta^{2}\left(A_{\mu}^{+}\right)_{\alpha}^{5}\left(A^{\mu}\right)_{5}^{\alpha} \\
& +\frac{1}{4} g_{5}^{2} v^{2}\left(A_{\mu}^{+}\right)_{\alpha}^{5}\left(A^{\mu}\right)_{5}^{\alpha}
\end{aligned}
$$

where the first term in the equation above is the kinetic energy of particle $\eta$, the second and third term are the tri-linear and quadri-linear interaction of particle $\eta$ with the gauge field, respectively, and the fourth term is the mass term of the gauge field, where the particle $W$ for $\alpha=1,2,3,4$ and particle $Z_{0}$ for $\alpha=5$, and they have the same mass: 


$$
m_{W}=m_{z}=\frac{1}{2} g_{5} v
$$

Experiments show that $m_{W} \sim m_{z} \sim 10^{2} \mathrm{GeV}$. This is the energy level of particle at the grand united point. If there is $g_{5} \sim 1$, then there is $v \sim 10^{2} \mathrm{GeV}$. Multiplying

$$
Z_{0}=-\frac{W}{\sqrt{2}}+\frac{3 B}{\sqrt{30}}
$$

in the Equation (7) by the normalizing factor $-\sqrt{5} / 2$ and rewriting

$$
\begin{aligned}
& Z_{0}=\cos \theta_{W} W+\sin \theta_{W} B \\
& \text { gives } \sin ^{2} \theta_{W}=\frac{3}{8}=0.375 .
\end{aligned}
$$

This value is significantly greater than the experimental value $\sin ^{2} \theta_{W}=0.23 \pm 0.015$. Later we will see that this is a result from neglecting gravity in the calculations above. We will obtain the result consistent with experiments if the gravity is included.

\section{Mass of Fermions}

The Higgs mechanism can give masses to quarks, electrons and neutrinos, too [2].

As the same as above, taking the unitary gauge gives

$$
\varphi^{\alpha}=\left(\varphi_{0}+\varphi^{\prime}\right)^{\alpha}=\frac{1}{\sqrt{2}}(v+\eta) \delta_{5}^{\alpha} .
$$

Using the Equation (2), the Yukawa coupling item of fermions with the Higgs field can be written as

$$
\sqrt{2} g^{\prime} \bar{\psi}_{\beta} \psi_{\alpha}^{\beta} \varphi^{\alpha}=g^{\prime}(v+\eta) \bar{\psi}_{\beta} \psi_{5}^{\beta}=g^{\prime}(v+\eta)\left(\bar{q} q+e^{+} e+v v\right),
$$

where $\bar{q} q=\sum_{i=1}^{3} \bar{q}_{i} q_{i}$. So, the quark, electron and neutrino have the same mass:

$$
m_{q}=m_{e}=m_{v}=g^{\prime} v .
$$

The undetermined constant $g^{\prime}$ could be determined if the quark mass is known. The equation above is logical, because the mass of the incident electron shrinks and the mass of the quark, especially the neutrino, increases significantly when the energy of the incident electron is transferred to the quark and neutrino. We know that the gravity between two particles is greatest when they have the same mass. Therefore the quark, electron and neutrino have the same mass at the grand unified point.

\section{The Unified Gauge Model Containing Gravity}

As mentioned previously, the reason why the theoretical value $\sin ^{2} \theta_{W}=0.375$ is greater than the experimental value $\sin ^{2} \theta_{W}=0.23 \pm 0.015$ is because gravity is neglected. How should be gravity introduced, then? 
In the physical course described by the Equation (1) there is also the positron $e^{+}$, neutrino $v_{e}$ and proton $p$ at the same time except the intermediate boson $W^{-}$; all of them are massive, so there is the gravity between them. Since the electron, neutrino has the same mass with quark and the proton $p(u u d)$ contains three quarks, the sum of masses of three particles $e^{+}, v_{e}$ and $p$ equals the mass of five quarks, and $W^{-}(\bar{u} d)$ contains two quarks. Suppose the gravity and the weak force on the intermediate boson are balanced at the grand unified point, there is

$$
5 A_{g}=-2 \cdot \frac{W}{\sqrt{2}},
$$

where $A_{g}$ is the gravitational potential of a quark and $-W / \sqrt{2}$ is the weak potential of a quark in the intermediate boson, so

$$
A_{g}=-\frac{2}{5} \cdot \frac{W}{\sqrt{2}} \text {. }
$$

Adding the equation above to the Equation (18) gives

$$
Z_{0}^{\prime} \equiv Z_{0}+A_{g}=-\frac{7}{5} \frac{W}{\sqrt{2}}+\frac{3 B}{\sqrt{30}} .
$$

Multiplying the equation above by the normalizing factor $-\sqrt{25 / 32}$ and rewriting

$$
\begin{gathered}
Z_{0}^{\prime}=\cos \theta_{W} W+\sin \theta_{W} B \\
\text { gives } \sin ^{2} \theta_{W}=\frac{15}{64} \approx 0.234 .
\end{gathered}
$$

This is consistent with the experimental value $\sin ^{2} \theta_{W}=0.23 \pm 0.015$. So, the total gauge field containing gravity is

$$
A_{\mu}(x)=\left(\begin{array}{ccccc}
S_{11} & S_{12} & S_{13} & \bar{X}_{1} & \bar{W}_{1} \\
S_{21} & S_{22} & S_{23} & \bar{X}_{2} & \bar{W}_{2} \\
S_{31} & S_{32} & S_{33} & \bar{X}_{3} & \bar{W}_{3} \\
X_{1} & X_{1} & X_{3} & X_{0}^{\prime} & W^{+} \\
W_{1} & W_{2} & W_{3} & W^{-} & Z_{0}^{\prime}
\end{array}\right),
$$

This is the unified gauge model to describe four interactions, where

$$
\begin{aligned}
& Z_{0}^{\prime} \equiv Z_{0}+A_{g}, \\
& X_{0}^{\prime} \equiv X_{0}-A_{g} .
\end{aligned}
$$

The interactive Lagrange density of fermions with the gauge field containing gravity

$$
L_{i}^{\prime}=g_{5} \bar{\psi} \gamma^{\mu} A_{\mu} \psi=g_{5} \bar{\psi} \hat{A}_{\mu} \psi
$$

is gauge invariant. When the scattered electron leaves the grand unified point, the $S U(5)$ group starts to break. When the electron leaves the nucleon, the strong interaction disappears, and when it is far away from the nucleon the weak interaction disappears, as shown in Figure 1. Thus, Equation (31) becomes 


$$
L_{i}^{\prime}=g_{e} e^{+} \hat{V}_{\mu} e^{-}+g_{g} v \hat{A}_{g} v \rightarrow 2 \gamma+2 v,
$$

This is consistent with Equation (1), where $\hat{V}_{\mu}$ is the 4-electromagnetic potential, and the gravitational coupling constant $g_{g}$ is far smaller than the electromagnetic coupling constant $g_{e}$.

\section{References}

[1] Weinberg, S. (1967) Physical Review Letters, 19, 1264.

Salam, A. (1968) In Elementary Particle Physics. Almqvist and Wilsell, Stokholm.

[2] Georgi, H. and Glashow, S.L. (1974) Physical Review Letters, 32, 438.

Hu, Y.-G. (1984) Gauge Field Theory. East China Normal University Press, Shanghai.

[3] Dai, Y.B. (2005) Interactional Gauge Theory. 2nd Edition, Science Press, Beijing. [In Chinese]

[4] Liang, B. (2015) Applied Physics Research, 7, 134.

[5] Liang, B. (2016) Applied Physics Research, 8, 96.

[6] Liang, B. (2016) Applied Physics Research, 8, 93.

[7] Liang, B. (2015) Journal of Modern Physics, 6, 982-989.

https://doi.org/10.4236/jmp.2015.67102

[8] Liang, B. (2018) Journal of Modern Physics, 9, 202-206.

https://doi.org/10.4236/jmp.2018.92013

[9] Fridman, J.I. (1972) Annual Review of Nuclear and Particle Science, 22, 203. https://doi.org/10.1146/annurev.ns.22.120172.001223

[10] Zhang, N.S. (1987) Particle Physics. 2nd Volume, Science Press, Beijing. (In Chinese) 